\title{
The interpenetration of two chain polymers in a good solvent
}

\author{
T. A. Witten, Jr. \\ Physique de la Matière Condensée College de France, Paris, France, \\ Physics Department, University of Michigan, Ann Arbor, Michigan 48109 \\ and Corporate Research Science Laboratories, Exxon Research and Engineering, Linden, New Jersey $07036^{\circ}$ \\ J. J. Prentis ${ }^{\mathrm{b})}$
}

Physics Department, University of Michigan, Ann Arbor, Michigan 48109

(Received 10 May 1982; accepted 28 June 1982)

\begin{abstract}
The interpenetration of two excluded-volume chain molecules of different size in dilute solution is studieci via scaling and renormalization methods. The chains are found to interpenetrate much more strongly than smoothed-density models suggest, in accordance with recent work by Khokhlov. The pair correlation funtion $g(r)$ goes to zero at the origin only as a weak power of $r$. This power is related to Des Cloizeaux's exponents $\theta$ describing intra chain correlations. The power is also related to the scaling exponents of star polymers. The mutual excluded volume $\boldsymbol{M}_{S L}$ of two chains with greatly different length is proportional to the volume of the smaller chain and to the mass of the larger. Thus $M_{S L}$ is much smaller than a smoothed density model would predict. We discuss which chain correlations give rise to this small $\mathrm{M}_{S L}$. The universal coefficient relating $M_{S L}$ to the radius of gyration of the smaller chain is strongly dependent on the dimension $d$ of space, according to our second-order expansion in $4-d$. The interpenetration behavior predicted here affects measurable thermodynamic, scattering, and physical-chemical properties of the solution.
\end{abstract}

PACS numbers: $61.25 . \mathrm{Hq}$

\section{INTRODUCTION}

Excluded-volume effects give rise to repulsion between long chain polymers dissolved in a good solvent. The repulsion increases the osmotic pressure, alters the light-scattering profile of the solution, and inhibits phenomena like chemical reactions requiring interchain contact. The interaction between two chains $S$ and $L$ can be described in terms of the correlation function $g_{S L}(r)$, a function proportional to the probability that the two chains are separated by a displacement $r$, and normalized to be 1 for $r \rightarrow \infty$. In a dilute solution containing $K$ polymers, each is excluded from a volume

$$
(K-1) M_{S L}=(K-1) \int d^{3} r\left[1-g_{S L}(r)\right]
$$

because of the others.

The properties of $g_{S L}(r)$ and of the excluded volume $M_{S L}$ have been discussed extensively. ${ }^{1-3}$ The methods of renormalized perturbation theory ${ }^{4-8}$ provide a systematic approach. In this article, we analyze the behavior of $g_{S L}(r)$ using scaling methods and renormalized field theory. The same scaling methods have been used recently by Khokhlov ${ }^{9}$ to predict the rate of certain chemical reactions.

A qualitative idea about the interchain correlations can be obtained by a smoothed-density approximation, ${ }^{1,10,3}$ in which each polymer (of $N$ monomers) is replaced by a uniform cloud of $N$ monomers with the same radius $R$. Then $g_{s L}(r)$ has the simple form $\exp [-U(r) / k T]$. The interaction energy $U$ of the overlapping densities $\rho_{S}$, centered at the origin, and $\rho_{L}$, centered at $r$, has the form $\beta \int d^{3} r^{\prime} \rho_{S}\left(r^{\prime}\right) \rho_{L}\left(r^{\prime}\right)$. For two

\footnotetext{
a) Present address and permanent address.

b) Horace H. Rackham, predoctoral fellow.
}

overlapping chains of different size the densities $\rho$ are roughly $N / R^{3}$, and the overlapping volume is order of the smaller radius cubed. Letting $S(L)$ be the number of monomers in the smaller (larger) chain, we have

$$
U(r) \sim R_{S}^{3} \frac{S}{R_{S}^{3}} \frac{L}{R_{L}^{3}} \sim S L^{1-3 \nu}
$$

where we have used the Flory relation $R \sim N^{\nu}, \nu \cong 0.6$. This energy goes to infinity as $S$ and $L$ go (together) to infinity, for any $r$ where the clouds overlap. Thus, the $g_{s}(r)$ for the two overlapping clouds is exponentially small in $N$ : the clouds behave as hard spheres. Their mutual excluded volume $M_{S L}$ varies as the sum of the radii cubed. If the two radii differ greatly, the larger one determines the excluded volume.

We find below that the chains interpenetrate much more strongly than this cloud model suggests. Indeed, $g_{S L}(r)$ decreases only as a weak power of $N$ or of $r$, even for $r \ll R$. And the mutual excluded volume $M_{S_{L}}$ for two chains of greatly different size becomes negligible compared to the volume of the large chain. In Sec. II we discuss the short distance $(r \ll R)$ behavior of $g_{S L}(r)$ for two chain ends and the analogous quantity $\hat{g}(r)$ for interior monomers. Section III gives an independent discussion of the mutual excluded volume $M_{S L}$ of a long chain and a short one. We relate $M_{S L}$ quantitatively to the short-chain radius using renormalization methods. In Sec. IV we discuss experimental tests of these predictions.

\section{INTERCHAIN CORRELATIONS AT SHORT DISTANCE}

We may readily express $g_{s_{L}}(r)$ in terms of partition functions of our system. We denote the partition function of a single chain by $Z_{S}$ or $Z_{L}$. We shall suppose one coordinate of the chain fixed at the origin, so as to 
eliminate a trivial factor of the solution volume. The probability that the two chains have endpoints separated by $r$ is proportional to the Boltzmann-weighted sum of all configurations of both chains with, say, an endpoint of $S$ at the origin and an endpoint of $L$ at $r$. We denote this sum as $Z_{S L}(r)$. The correlation function $g_{S L}(r)$ may be written $g_{S}(r)=Z_{S L}(r) /\left(Z_{S} Z_{L}\right)$.

There are two characteristic length scales governing $g_{s i}(r)$ : the monomer length scale $h$ and the size $R$ of the chain. This $R \rightarrow \infty$ with the chain length, while $h$ remains fixed. The dimensionless function $g_{s}(r)$ depends only on ratios of lengths; e.g. ,

$$
g_{S L}(r)=f_{1}\left(r / h, r / R_{S}, R_{L} / R_{S}\right) \text {. }
$$

(The ratios $R / h$ and thence $N$ are a combination of these arguments. The monomer excluded volume $\beta$ will be held fixed on the scale of $h$ in our discussion.)

For $r, R \gg h, g_{s L}$ must have a finite limit, and is expressible in terms of macroscopic variables only:

$$
g_{S L}(r) \underset{r, R_{S}, R_{L} \gg h}{\longrightarrow} f_{2}\left(r / R_{S}, R_{L} / R_{S}\right) \text {. }
$$

We obtain further information about $g_{s_{L}}(r)$ by considering the limit $r=h$, and specializing to a lattice selfavoiding walk with lattice spacing $h$. Then, as Khohlov noted, ${ }^{9} Z_{S_{L}}(r)$ becomes the partition function of a single chain of length $S+L+1$, with two adjacent links in fixed positions. This must have the same $N \rightarrow \infty$ behavior as that of an unrestricted chain ${ }^{11}$ of that length: $Z(N)$ $\sim N^{\gamma-1} \tau_{c}^{N}$, where. $\gamma$ is a universal critical exponent and $\tau_{c}$ a nonuniversal constant. Thus,

$$
Z_{S L}[r=O(h)] \sim(S+L)^{r-1} \tau_{c}^{S+L} .
$$

And

$g_{S L}[r=O(h)] \sim\left\{\frac{S+L}{S L}\right\}^{\gamma-1} \sim R_{S}^{-(\gamma-1) / \nu}\left[\left(R_{S} / R_{L}\right)^{1 / \nu}+1\right]^{\gamma-1}$.

We expect this to be compatible with the scaling form Eq. (3) over some range of $r$. This leads to

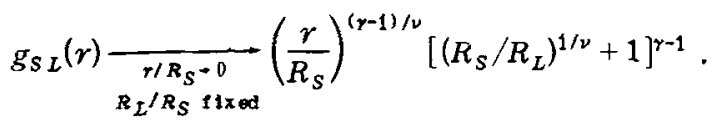

Thus $g_{S L}(r)-0$ for small $r$, as in the smoothed density model discussed above. But it does so with a relatively weak power-law dependence, rather than an exponential decrease.

When one chain is much shorter than the other, it is the shorter chain which determines $g_{S L}(r)$ at small $r$. If $R_{L} / R_{S} \rightarrow \infty$ in Eq. (5) above, $g_{S L}(r)$ becomes independent of the larger $R_{L}$ for $r \ll R_{S}$. Over the wider range $R_{S} \leqslant r \ll R_{L}, g_{S L}(r)$ should remain independent of $R_{L}$. On the one hand, $g(r)$ should increase monotonically with $r$, even as $R_{L} \rightarrow \infty$. On the other hand, $g(r)$ for fixed $r$ must decrease with increasing $R_{L}$. In view of the nonzero limit for small $r$ [Eq. (5)] $g(r)$ must remain nonzero for larger $r$. Thus,

$$
\lim _{\substack{R_{L} \rightarrow \infty \\ R_{S},+1 \text { ixed }}} g_{S L}(r)
$$

must be a nonzero function of $r$. The integral of $g_{S L}$ must give the excluded volume $M_{S L}$. The linear be- havior of $M_{S L}$ with $L$, derived below, implies that $\left(1-g_{S L}\right)$ falls off as $r^{1 / v-d}$ or faster for $R_{S} \ll r \ll R_{L}$, in $d$-dimensional space. This power is natural, since it also describes ${ }^{12,13}$ the average density $\rho_{L}$ of $L$ chain monomers at distances $r \ll R_{L}$ from an end of the isolated $L$ chain. In this $r$ regime the repulsion effect may apparently be regarded as a weak perturbation, linear in $\rho_{L}$.

The behavior of $g_{S L}$ inferred here is based on the known behavior of the two-chain partition function for small $r$. It may also be derived from properties of a single chain as noted by Khohlov. ${ }^{9}$ We let $Z_{S}(r)$ represent the partition function for a single chain whose ends are separated by the displacement $r$. One may infer ${ }^{14}$ the short-distance behavior of $Z_{S}(r)$ directly from its renormalized form. The result is the same power law obtained above for $g_{S L}$; viz.,

$$
Z_{S}(r)=Z_{s}\left(r_{0}\right)\left(r / r_{0}\right)^{(\gamma-1) / \nu}
$$

One obtains this $r$ dependence by fixing the reference distance $r_{0}$ and calculating $Z_{s}(r) / Z_{s}\left(r_{0}\right)$. The above power law arises from the repulsion between monomers chemically near the ends; it is derived without mention of the chain length $S$; thus $S$ may be taken as infinite. The fact that the two ends belong to a single chain is evidently irrelevant in the calculation:

$$
\frac{Z_{S}(r)}{Z_{S}\left(r_{0}\right)}=\frac{Z_{S L}(r)}{Z_{S L}\left(r_{0}\right)}=\frac{g_{S L}(r)}{g_{S L}\left(r_{0}\right)}
$$

Thus again we must have $g_{S L}(r) \sim r^{(\gamma-1) / \nu}$.

The small- $r$ behavior deduced above relies on the requirement that the two points separated by $r$ be endpoints of chains. One may also treat short-distance correlations of arbitrary points along the chains. We consider a point on the $S$ chain at some distance $N_{1}$ from one end and $N_{2}$ from the other. We choose a corresponding point on chain $L$ at a distance $N_{3}, N_{4}$ from the two ends. We then define $\hat{g}(r)$, analogous to $g_{S L}(r)$, giving the relative probability that the two specified points are separated by a displacement $r$. We expect $\hat{g}(r)$, like $g_{S L}(r)$, to have a characteristic power behavior for short distances $r h \leqslant r \ll R_{1} \cdots R_{4}$. As with $g_{S L}$, one may deduce this $r$ dependence from the correlations of the two monomers in a single chain formed, e.g., by joining the ends ${ }^{9}$ of segments 2 and 3 . Des Cloizeaux ${ }^{14}$ has solved this problem approximately using renormalized field theory. The result has the same structure as do the end-end correlations. For small $r$ the correlation function has a power-law behavior: $\hat{g}(\gamma)$ $\sim r^{\theta_{2}}$, where $\theta_{2} \sim 0.71$. For $r$ in the intermediate range $R_{S} \ll r \ll R_{L}$, we expect that in $d$ dimensions,

$$
(1-\hat{g}) \sim\left(r / R_{S}\right)^{1 / v-d}
$$

as with $g_{S L}$.

This $\hat{g}(r)$ controls the scaling of star polymers. We may express it as a ratio of partition functions as with $g_{S L}(r)$ :

$$
\hat{g}(r)=\hat{Z}(r) / \mathcal{Z}_{S} \mathcal{Z}_{L},
$$

where $\hat{Z}(r)$ is the partition function of the two chains with the two chosen points constrained to lie at displace- 
ment $r$ apart. Thus when $r=h, \hat{Z}(r)$ is the partition function of a four-branched star polymer, up to a factor independent of the $N_{i}$. This partition function should scale with the $N_{i}$ in a way analogous to the scaling of a single chain, as noted by Daoud and Cotton ${ }^{15}$

$\hat{Z}[r=O(h)] \sim N_{1}^{A_{4}} \tau_{c}^{N_{1}+N_{2}+N_{3}{ }^{+N} 4} f_{3}\left(N_{2} / N_{1}, N_{3} / N_{1}, N_{4} / N_{1}\right)$.

Repeating the reasoning used above for $g_{S L}(r)$, we find

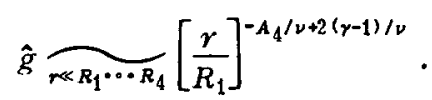

Comparing with $\hat{g}(r) \sim r^{\theta} 2$; we have $\mathrm{e}^{16}$

$$
A_{4}=2(\gamma-1)-\nu \theta_{2} \text {. }
$$

Using ${ }^{17} \gamma=1.162$ and $\nu=0.588$ one finds $A_{4} \cong-0.1$. To obtain the scaling exponent $A_{3}$ of 3 -branched stars, one replaces $\theta_{2}$ by Des Cloizeaux's $\theta_{1} \cong 0.46$; this gives $A_{3} \cong+0.05$. These predictions appear at odds with those of Daoud and Cotton ${ }^{15}$ for many-branched stars.

\section{MUTUAL EXCLUDED VOLUME}

Either $g_{s L}(r)$ or $\hat{g}(r)$ may be integrated to obtain the mutual excluded volume $M_{S L}$. In the excluded-volume limit $M_{S L}$ scales as the volume $R^{d}$ of the chains if both $S$ and $L$ go to $\infty$ in proportion. ${ }^{18,19}$ Thus,

$$
M_{S L}=R_{S}^{d} f_{4}(L / S), L, S \gg 1 \text {. }
$$

One may anticipate the behavior of $f_{4}$ by examining a limiting case. For $S=O(1)$ and $L \rightarrow \infty$, the repulsive energy $U(r)[\mathrm{Eq} .(1)]$ goes to 0 , the $S$ chain completely penetrates the $L$ chain region, and $M_{S L} \sim U(r) R_{L}^{d} \sim L$. This linear prediction, originally made by de Gennes, ${ }^{20}$ contradicts the cloud model and several proposals in the recent literature. ${ }^{7,18}$ At issue is the question whether two chains with a given large $L / S$ interact the same whether $S=O(1)$ or $S \gg 1$. In the cloud model, the two cases behave quite differently. If $S=O(1)$ the $S$ cloud penetrates the $L$ cloud easily, but if $S \gg 1$ the $S$ cloud is excluded from the $L$ cloud. Below we extend the de Gennes argument to show that $M_{S L} \sim L$ for two chains even when $S \gg 1$.

We note first that two chains of equal length $S$ have a mutual excluded volume $M_{s s}$ proportional to $R_{s}^{d}$. The long chain may be thought of as being composed of $\lambda$ $=L / S$ segments of length $S$ apiece. First we consider, instead of the $L$ chain, a cloud of these $\lambda$ segments confined to the volume $R_{L}^{d}$ normally occupied by the $L$ chain. We also remove the excluded volume interactions between the various $\lambda$ segments, while retaining it for monomers within a segment. This $L$ cloud is a dilute solution of the segments -indefinitely dilute as $\lambda \rightarrow \infty$. Thus the mutual excluded volume between chain $S$ and the cloud is simply $\lambda$ times the excluded volume of two $S$ chains. This is true whether the $\lambda$ segments are free to move or are fixed at definite positions within the cloud. We may, e.g., fix the end position of each $\lambda$ segment without affecting the mutual excluded volume. This would not hold if both chains were broken into many segments. Then if the segments are mobile in their respective clouds, the mutual excluded volume is of or$\operatorname{der}\left(R_{S}+R_{L}\right)^{\natural}$, while if the segments are fixed, it is merely the sum of segment volumes.
Accordingly we position the end of each $\lambda$ segment at the end of its predecessor to reconstruct the $L$ chain. Finally, we restore the repulsion between the monomers of different $\lambda$ segments. This has two effects. It pushes the segments away from one another; it also stretches out each segment. Neither of these effects changes the excluded volume qualitatively. The first only makes the initial approximation of the separated segments more nearly valid. The second effect is the distortion of a segment by the attachment of a long excluded-volume tail to each end. Now, it is known that the attachment of such tails changes the average separation of two monomers by only a finite factor, even for infinitely long tails. ${ }^{14,21}$ Thus, the density of monomers within a fixed distance such as $R_{S}$ of a segment is changed by only a finite factor when the intersegment repulsions are turned on.

The reconstruction of the $L$ chain is now complete. The mutual excluded volume $M_{S L}$ remains of order $\lambda$ times that of the two $S$ chains. Thus $M_{S L}$ is linear in the longer chain length, as claimed.

The methods of renormalized perturbation theory allow us to estimate the magnitude of $M_{S L}$ in several ways. These methods rely on an expansion in $\epsilon \equiv 4-d$. The calculations of Knoll, Schäfer, and Witten, ${ }^{18}$ or Elderfield ${ }^{7}$ or Oono and Freed ${ }^{22}$ give $M_{S L} / M_{S S}$ in terms of $S$ and $L$. Such calculations give logarithms of $S$ and $L$ instead of the desired powers. By requiring ${ }^{22}$ that these logarithms be consistent with the form $M_{S L} / M_{S S}$ $\overrightarrow{L \gg S} c(\epsilon) L / S$, one finds $c(\epsilon)=1+\epsilon / 8-\epsilon(\ln 2) / 2+O\left(\epsilon^{2}\right)$.

One may obtain another quantitative measure of $M_{S_{L}}$ in terms of the volumes $R^{d}$ of the chains. We calculate below the amplitude $\alpha$ defined by

$$
M_{S L} \underset{L \gg S}{\longrightarrow} \alpha R_{S}^{d} L / S \text {, }
$$

where $R_{S}$ is the radius of gyration of the $S$ chain, using the method of Ref. 23. In this method one renormalizes the partition functions of a grand canonical ensemble ${ }^{24}$ of chain lengths. The canonical results are then obtained by an exact closed-form transformation. ${ }^{25}$

The excluded volume $M_{S L}$ may be expressed in terms of $Z_{S}, Z_{L}$, and $Z_{S L}(r)$ :

$$
M_{S L}=\frac{-\int d^{d} r\left[Z_{S L}(r)-Z_{S} Z_{L}\right]}{Z_{S} Z_{L}}
$$

These partition functions have long-chain limiting forms as given above Eq. (4). For the individual chains,

$$
Z_{s}=f_{0} S^{\gamma-1} \tau_{c}^{s} ; Z_{L}=f_{0} L^{\gamma-1} \tau_{c}^{L},
$$

where $f_{0}$ is a nonuniversal amplitude. The scaling of $\int d^{d} r\left[Z_{S L}(r)-Z_{S} Z_{L}\right]$ may be inferred from the finiteness of $\alpha$ :

$$
\int d^{d} r\left[Z_{S L}(r)-Z_{S} Z_{L}\right]=f_{S L} S^{(\gamma-1)+\nu d-1} L^{\gamma} \tau_{c}^{S+L}
$$

for $L \gg S$. The radius of gyration $R_{S}$ can be expressed ${ }^{23}$ as a ratio of partition functions $Y_{S}(q)$ :

$Y_{S}(q) \equiv \frac{1}{2} \sum_{\left\{r_{i}\right\}} \sum_{i, j} \exp \left[i q \cdot\left(r_{i}-r_{j}\right)\right] \exp \left[-E\left\{r_{i}\right\} / k T\right]$, 
where $r_{i}$ is the position of the $i$ th monomer, $\left\{r_{i}\right\}$ is a configuration of the chain, $E\left\{r_{i}\right\}$ is the repulsive energy of a configuration, and $r_{0}=0$. The structure function $S(q)$ for a chain is simply $Y_{S}(q) / Z_{s}$; hence $R_{s}$ can be expressed in terms of the amplitudes $y_{0}$ and $y_{2}$ of the Taylor expansion of $Y(q)$ :

$$
\begin{aligned}
Y(q) & =N^{\gamma-1+2} \tau_{c}^{N}\left(y_{0}-y_{2} N^{2 \nu} q^{2}+\cdots\right) \\
& =\text { const. } S(q)=\text { const. }\left[1-R^{2} q^{2} / d+O\left(q^{4}\right)\right] .
\end{aligned}
$$

Evidently, $R^{2} / d=y_{2} / y_{0}$. The ratio $\alpha$ may now be written

$$
\alpha=\frac{-f_{S L}}{f_{0}^{2}\left(y_{2} / y_{0}\right)^{d / 2} d^{d / 2}} .
$$

Each of the partition functions $Z, Y$, may be expressed in terms of its grand canonical counterpart. Here the $S$ and $L$ chains are each taken from a grand canonical ensemble of chain lengths, governed by monomer fugacities $\tau_{S}^{-1}$ and $\tau_{L}^{-1}$. The grand canonical partition function for the short chain, $Q_{1}\left(\tau_{S}\right)$, is related to the $Z(N)$ by

$$
Q_{1}\left(\tau_{s}\right) \equiv \sum_{N=0}^{\infty} \tau_{s}^{-N} Z(N),
$$

and similarly for chain $L$.

Each of the amplitudes $f, y$ may be readily expressed in terms of its counterpart $\tilde{f}, \tilde{y}$ in the grand canonical ensemble. Thus

$$
Q_{1}\left(\tau_{s}\right) \equiv \tilde{f}_{0} t_{s}^{-\gamma}, \text { where } \tilde{f}_{0}=f_{0} \Gamma(\gamma),
$$

and where $t_{S} \equiv \ln \left(\tau_{S} / \tau_{c}\right)$. This limiting relation between the large $-N$ behavior and the $\tau \rightarrow \tau_{c}$ behavior holds whenever the $t$ power is negative. Using this transformation law, valid also for $Z_{S L} \rightarrow Q_{S L}$, the ratio $\alpha$ may be expressed in terms of the amplitudes $\bar{f}, \bar{y}$ in the combination $\bar{\alpha}$.

$$
\alpha=\frac{\Gamma(\gamma)}{\gamma \Gamma(\gamma+\nu d-1)}\left[\frac{\Gamma(\gamma+2+2 \nu)}{\Gamma(\gamma+2)}\right]^{\alpha / 2} \bar{\alpha},
$$

where

$$
\tilde{\alpha} \equiv \frac{-\tilde{f}_{S L}}{\bar{f}_{0}^{2}\left(\tilde{y}_{2} / \tilde{y}_{0}\right)^{d / 2} d^{d / 2}} .
$$

The grand canonical ratio $\bar{\alpha}$ may be readily calculated by the renormalized Fixman expansion. ${ }^{23}$ The Appendix outlines our calculation to second order in $\epsilon$ (one-loop order). We obtain

$$
\bar{\alpha}=2^{d-1} \pi^{d / 2} \Gamma(d / 2) d^{d / 2} \frac{1}{4} \epsilon[1+(85 / 96) \epsilon] .
$$

In view of the sizeable $\epsilon^{2}$ correction, we estimate a factor-of-two uncertainty in this result in three dimensions.

The combination of $\Gamma$ functions $\alpha / \bar{\alpha}$ may be calculated accurately using the accepted ${ }^{17}$ values of $\gamma$ and $\nu$; one finds $\alpha / \tilde{\alpha}=6.569$ in three dimensions. Combining this with our expression for $\bar{\alpha}$, [Eq. (23)] we conclude that for $L \gg S$ and $d=3$,

$$
\begin{aligned}
M_{S L} & =(11.76 \pm 5.5) L / S R_{S}^{3}, \\
& \cong 2.8 L / S\left[4 \pi / 3 R_{S}^{3}\right] .
\end{aligned}
$$

This expression for $M_{S I}$ allows us to make a new estimate for the interpenetration function ${ }^{1} \psi$ for a monodis- perse solution of excluded-volume chains in three dimensions:

$$
\begin{aligned}
\psi & \equiv \frac{1}{4 \pi^{3 / 2}} \frac{M_{S S} / 2}{R_{S}^{3}}, \\
& =\frac{1}{4 \pi^{3 / 2}} \frac{1}{2}\left[\frac{M_{S S} L}{M_{S L} S}\right] \alpha .
\end{aligned}
$$

The constant in square brackets is the inverse of $c(\epsilon)$ defined above [Eq. (13)]. Combining this with our expression for $\alpha$ from Eqs. (21) and (23), we obtain $\psi=0.14 \epsilon[1$ $+1.11 \epsilon]=0.30 \pm 0.16(\epsilon=1)$. The recent experiment of Miyaki et al. ${ }^{26}$ suggests $\psi=0.22$, which is consistent with this result. These coefficients [Eqs. (24) and (25)] will vary from their asymptotic values if the solvent is worsened or the chains shortened. A calculation of these variations is in progress. ${ }^{27}$

\section{EXPERIMENTAL TESTS}

There are two classic experiments which are sensitive to the excluded volume $M_{S_{L}}$ : osmotic pressure and light scattering. In a solution containing $S$ and $L$ chains with chain concentrations $c_{S}$ and $c_{L}$, the osmotic pressure II has the form

$$
\begin{aligned}
\frac{\Pi}{k T}= & c_{S}+c_{L}+\frac{1}{2} M_{S S} c_{S}^{2} \\
& +M_{S L} c_{S} c_{L}+\frac{1}{2} M_{L L} c_{L}^{2}+O\left(c^{3}\right) .
\end{aligned}
$$

The scattering structure function $S(q)$ for $q=0$ has a similar form. Schäfer ${ }^{28}$ has recently reviewed the available experimental information on $M_{S L}$. It appears from this review that the experiments are not yet precise enough to confirm or disprove our prediction. Indeed, they do not even rule out the hard sphere behavior predicted by the smoothed density hypothesis.

The short-distance behavior of the interchain correlation function $g_{S L}(r)$ is measurable in neutron scattering experiments. By marking certain monomers $i, j$ isotopically one may measure the structure function $S(q)$ conjugate to the correlation function $g_{i j}(r)$. By marking only monomers near one end of the chains, one may thus measure $g_{S L}(r)$. The power-law behavior of $g_{S L}(r)$ for short distances produces a conjugate power-law behavior in $S(q)$. Thus, in a dilute solution for wavelengths $2 \pi / q$ much longer than the size $R_{m}$ of the marked sections yet much shorter than the chain radius $R$, one expects

$$
S(q)=N_{m}^{2} c\left[1+c a_{2} q^{[-(\gamma-1) / \nu]-d}\right],
$$

where $c$ is the chain concentration and $N_{m}$ is the number of markers per chain. By marking $N_{m}$ monomers near the middle of the chains, one should find analogous behavior, with $(\gamma-1) / \nu$ replaced by $\theta_{2}$. This same behavior should occur in the semidilute regime, provided $1 / q$ is small compared to the screening length $\xi$. The constant $a_{2}$ should then be replaced by a function of the overlap $c R^{3}$. If the wavelength becomes comparable to the size of marked segment, the formula should be multiplied by $S_{m}(q)$, the form factor of the one marked segment. This too may be a function of concentration in the combination $c R_{m}^{3}$. These predictions are not altered by polydispersity of the solution, provided all chains 
are much longer than the scattered wavelength $1 / q$. The same powers of $q$ predicted here could have been observed in principle by marking, e.g. , the two ends of the same chain. The predicted power has not to our knowledge been seen. It may prove easier to see the interchain effects predicted here, since (a) it is easier to make a long polymer with one marked section than with two, and (b) the interchain scattering can be readily extracted, since it has a special concentration dependence.

There exist spectroscopic probes sensitive to the concentration of nearby pairs of monomers. Thus, e.g. , one may detect the number of ends separated by some small distance $b$ by measuring the optical absorption due to some hybridized excited state of the two ends. The number of such pairs is of order $c^{2} g_{s L}(b)$. The number of pairs thus has the characteristic $S$ and $L$ dependence of Eq. (4), noted by Khohlov, provided the solution is dilute, $c \ll R^{-3}$. The probed ends need not be the same as the other monomers. The method could also be used to measure the contacts between the two ends of the same chain. Experiments observing the expected powers have not been reported.

Clearly $g_{s L}(r)$ also influences chemical or isomeric reactions between two chain ends. Here also the reaction rate is proportional to the number of pairs within some microscopic distance $b$ of each other. Khohlov ${ }^{9}$ noted that such reactions, if "chemically limited," have a rate proportional to $g_{S L}(r \cong b) \sim N^{\gamma-1}$. The present work extends these results to cover the region $b>1$. The chemically limited restriction is a severe one, since here the reactive sites are necessarily dilute. If the reaction is started at time $t=0$, it will be chemically limited only for short times. That is, if two reactants already within the distance $b$ react in a mean time $t_{0}$, then the reaction ceases to be chemically limited for times greater than $t_{0}$. After this time, the reaction becomes transport limited; the number of nearby pairs is then much depleted compared to the equilibrium number. The behavior of the reaction rate in this case is a subtle one ${ }^{29-30}$-even in a melt, where there are no excluded volume effects of the sort considered here. To generalize the reaction-rate theory to treat excluded volume effects would be valuable.

This work benefited greatly from discussions with P. G. de Gennes and Lothar Schäfer. Professor Lothar Schäfer suggested several valuable improvements in the manuscript. Ludvik Leibler pointed out Khokhlov's work to us. T.W. is grateful for hospitality and support to the Condensed Matter Physics department of Collège de France, where most of this work was done. Supported in part by the U. S. National Science Foundation under DMR 80-12867, polymer program, and by the French CNRS.

\section{APPENDIX: CALCULATION OF $\bar{\alpha}$}

The Laplace transform of Eq. (16) is the connected part of the two-chain partition function which we denote $Q_{2_{c}}$. Its Fixman expansion to second order in the repulsion strength $u$ is represented by the diagrams of Fig.

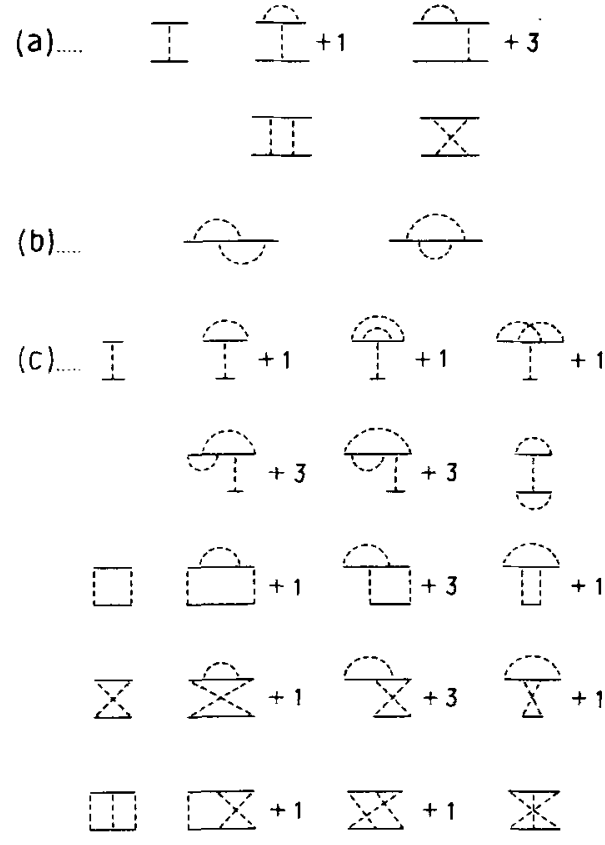

FIG 1. Perturbation diagrams used in calculating $\alpha$. (a) Diagrams for $Q_{2 c}$ up to second order. The notation $+1,+3$ indicates one or three other diagrams related to the one shown by interchanging the two chains or their ends. (b) Diagrams for $Q_{1}$ giving rise to the $z_{0}$ renormalization. (c) Diagrams for $\Gamma^{(4)}$ giving rise to the $z_{4}$ renormalization.

1(a). Evaluating these gives

$$
\begin{aligned}
Q_{2_{c}}= & \frac{K^{2}(2 \pi)^{d} u}{t_{S}^{2} t_{L}^{2}}\left\{1-2 u\left[I\left(0, t_{S}\right)+I\left(0, t_{L}\right)\right]\right. \\
& \left.+u\left[I\left(t_{S}, t_{S}\right)+I\left(t_{L}, t_{L}\right)\right]+2 u I\left(t_{S}, t_{L}\right)\right\},
\end{aligned}
$$

where

$$
\begin{aligned}
I\left(t_{S}, t_{L}\right) & \equiv \int d^{d} p \frac{1}{\left(t_{S}+p^{2}\right)\left(t_{L}+p^{2}\right)}, \\
& =\frac{\Xi(d)}{2} \frac{\Gamma(d / 2) \Gamma(\epsilon / 2)}{1-\epsilon / 2} \frac{t_{S}^{1-\epsilon / 2}-t_{L}^{1-\epsilon / 2}}{t_{S}-t_{L}},
\end{aligned}
$$

where $\Xi(d) \equiv 2 \pi^{d / 2} / \Gamma(d / 2)$ is the area of the unit sphere in $d$ dimensions. The constant $K$ enters through the free-chain partition functions $Q_{0}(t, p)$ in the expansion: $Q_{0}(t, p) \equiv K /\left(p^{2}+t\right)$. Each solid line segment in the diagrams denotes a factor $Q_{0}$. The repulsion strength $u$ is proportional to the excluded volume of a monomer pair. The values of $K$ and $u$ for a lattice polymer are given in Ref. 23. To the first nonleading order in $u$, the singlechain partition function $Q_{1}$ has the form

$$
Q_{1}(t)=(K / t)[1-u I(0, t)] .
$$

To renormalize $Q_{2_{c}}$ and $Q_{1}$ we define a renormalization length scale $\chi^{-1}$, define a dimensionless $t$ by $\hat{t} \equiv t \chi^{-2}$ and then express $I\left(t_{s}, t_{L}\right)$ :

$$
I\left(t_{S}, t_{L}\right)=\Xi(d) \chi^{-\epsilon}\left[\frac{1}{\epsilon}+\hat{I}\left(\hat{t}_{S}, \hat{t}_{L}\right)\right],
$$

the regular part $\hat{I}$ being analytic in $\epsilon$ for small $\epsilon$ :

$$
\hat{I}\left(\hat{t}_{S}, \hat{t}_{L}\right)=-\frac{1}{2} \frac{\hat{t}_{S} \ln \hat{t}_{S}-\hat{t}_{L} \ln \hat{t}_{L}}{\hat{t}_{S}-\hat{t}_{L}}+O(\epsilon) .
$$


We then change to rescaled variables $t \rightarrow t_{R} \equiv z_{2} t, u \rightarrow g$ $\equiv z_{4} \Xi(d) u \chi^{-\epsilon} ; K \rightarrow K_{R} \equiv z_{0} K$, choosing $z_{2}$ and $z_{4}$-independent of $t_{S}, t_{L}$-to remove the singular $1 / \epsilon$ terms of $Q_{1_{R}}\left(t_{R}, g\right) \equiv z_{0} Q_{1}\left(t_{R}, g\right)$. We specify these in more detail below.

From the expression for $z_{4}$ to second order in $g$, one calculates

$$
\frac{\partial g}{\partial \ln \chi}=g\left[-\epsilon-4 g-\frac{21}{2} g^{2}\right] .
$$

In the limit of large polymers, hence small $\chi, g$ is . driven to the fixed point $g^{*}$ defined by the vanishing of this derivative. Thus

$$
g^{*}=-\left[\frac{\epsilon}{4}+\frac{21}{128} \epsilon^{2}+O\left(\epsilon^{3}\right)\right] .
$$

The "renormalized" expansion of $Q_{1_{R}}$ in terms of $t_{R}$ and $g$ has the same form as the unrenormalized expansion, but with all the singular inverse powers of $\epsilon$ removed from the $I$ integrations.

The renormalization or scaling symmetry of $Q_{1}$ and $Q_{2_{c}}$ follows from the invariance of the physical partition functions when the renormalization scale $\chi$ is changed. The scaling laws are ${ }^{6,19}$

$$
\begin{aligned}
& Q_{2_{c R}}\left(t_{S R}, t_{L R}\right)=t_{S R}^{-2 \gamma-\nu t} Q_{2_{c R}}\left(1, t_{L R} / t_{S R}\right) ; \\
& Q_{1_{R}}\left(t_{R}\right)=t_{R}^{-\gamma} Q_{1_{R}}(1) .
\end{aligned}
$$

Thus the mutual excluded volume

$$
\begin{aligned}
& \frac{Q_{2_{G R}}\left(t_{S R}, t_{L R}\right)}{\left[Q_{1_{R}}\left(t_{S_{R}}\right) Q_{1_{R}}\left(t_{L R}\right)\right]}=\frac{t_{S R}^{-\nu d}}{r} \frac{g^{*}(2 \pi)^{d}}{\Xi(d)} \\
& \quad \times\left[1+g^{*}\left(-1+\frac{r \ln r}{1-r}\right)+O\left(g^{* 2}\right)+O\left(\epsilon g^{*}\right)\right] .
\end{aligned}
$$

Here we have evaluated the integrals at $d=4$ and denoted $t_{L} / t_{S}\left(=t_{L R} / t_{S R}\right)$ as $r$. The ratio $\tilde{y}_{2_{R}} / \bar{y}_{0_{R}}$ of Eq. (22) was calculated in Ref. 23 with the same renormalization conventions:

$$
\tilde{y}_{2_{R}} / \tilde{y}_{0_{R}}=\left(1+\frac{\epsilon}{96}\right) t_{R}^{-2 \nu}
$$

We combine these results to form $\bar{\alpha}$ :

$$
\tilde{\alpha} \equiv \lim _{t_{L} / t_{S}{ }^{-0}} \frac{-t_{L} / t_{S} Q_{2_{c}}\left(t_{S}, t_{L}\right)}{Q_{1}\left(t_{S}\right) Q_{1}\left(t_{L}\right)\left[\tilde{y}_{2} / \tilde{y}_{0}\right]^{d / 2} d^{d / 2}} .
$$

This quantity is invariant under the scale factors $z_{0}$, $z_{2}$, and $z_{4}$; thus it can be expressed in terms of the renormalized ratio of Eqs. (A9) and (A10) above:

$\bar{\alpha}=\lim _{r \rightarrow 0} \frac{-g^{*}(2 \pi)^{d}}{\Xi(d)(1+\epsilon / 96)^{d / 2} d^{d / 2}}\left[1+g^{*}\left(-1+\frac{r \ln r}{1-r}\right)\right]$.

Evidently, the term in $\ln r$ drops out as $r \rightarrow 0$. We choose to factor out the kinematic factor $(2 \pi)^{d} d^{d / 2} / \Xi(d)$ and make a strict $\epsilon$ expansion of the rest. Inserting Eq. (A6) for $g^{*}$ this gives Eq. (23) in the text:

$$
\tilde{\alpha}=2^{d-1} \pi^{d / 2} \Gamma(d / 2) d^{d / 2} \frac{\epsilon}{4}\left[1+\frac{85}{96} \epsilon\right] \text {. }
$$

We now give the renormalization prescription defining the $z_{i}$ factors. The factor $z_{2}$ renormalizing $t$ is defined as described in Ref. 23. The renormalization of $K$ (not needed in Ref. 23) is required beyond first order in $g$ to render the fixed-end partition function $Q_{1_{R}}(p, t=0)$ free of an overall multiplicative divergence in inverse powers of $\epsilon$. To second order in $g$ one has

$$
\begin{aligned}
& Q_{1_{R}}(p, t=0) \\
& \quad=z_{0} K\left\{\frac{1}{p^{2}}+\frac{g^{2} \chi^{2} \epsilon}{p^{2}}\left(p^{2-2 \epsilon}\left[-\frac{1}{(8 \epsilon)}-\frac{9}{32}+O(\epsilon)\right]\right) \frac{1}{p^{2}}\right\},
\end{aligned}
$$

represented by the two diagrams of Fig. 1(b). Evidently, the choice $z_{0}=1+g^{2} /(8 \epsilon)$ removes the singular part.

The factor $z_{4}$ is chosen to render finite those diagrammatic pieces (called collectively $\Gamma_{R}^{(4)}$ ) which if replaced by an interaction line would yield a valid lower-order diagram. These $\Gamma_{R}^{(4)}$ diagrams are pictured in Fig. 1c. We may determine the divergent part by evaluating these at the "symmetry point" $S P_{x}$, where the sum of any two external momenta $p_{i}$ has magnitude $\chi$. An overall factor $z_{0}^{-2}$ enters, because $K$ has been expressed in terms of $K_{R}$ in the $Q_{0}$ factors. $^{31}$

$$
\begin{aligned}
\left.\Gamma_{R}^{(4)}\right|_{S P_{\mathrm{X}}}= & z_{0}^{-2}\left[g z_{4}^{-1}+4\left(g z_{4}^{-1}\right)^{2} I_{1}(k)\right. \\
& \left.+5\left(g z_{4}^{-1}\right)^{3}\left[I_{1}(k)\right]^{2}+22\left(g z_{4}^{-1}\right)^{3} I_{4}(k, k)\right]_{k=1} .
\end{aligned}
$$

Here $k$ is the momentum of a pair of external lines, expressed as a multiple of $\chi$. The integrals $I_{1}$ and $I_{4}$ are convolutions of two or of three propagators, and are defined in Amit (Ref. 31) Eqs. A9-11 and A9-35. Expanded in $\epsilon$, these are

$$
\begin{aligned}
& \left.I_{1}(k)\right|_{k=1}=\frac{1}{\epsilon}\left[1+\frac{1}{2} \epsilon\right]+O(\epsilon) ; \\
& \left.I_{4}(k, k)\right|_{k=1}=\frac{1}{2 \epsilon^{2}}\left[1+\frac{3}{2} \epsilon\right]+O\left(\epsilon^{0}\right) .
\end{aligned}
$$

One determines $z_{4}^{-1} \equiv 1+a_{1} g+a_{2} g+\cdots$ to cancel the inverse powers of $\epsilon$ appearing in Eq. (A15) for $\Gamma_{R}^{(4)}$. As in Ref. $23 a_{1}=-4 / \epsilon$ removes the divergence in order $g^{2}$. The choice $a_{2}=16 / \epsilon^{2}-21 /(4 \epsilon)$ removes them in $O\left(g^{3}\right)$. Based on these values for $a_{1}$ and $a_{2}$, one may now calculate

$$
\frac{\partial g}{\partial \ln \chi}=-\epsilon g\left[1-\frac{\partial \ln z_{4}}{\partial \ln g}\right]^{-1} \text {. }
$$

This yields Eq. (A5) above.

${ }^{1} \mathrm{H}$. Yamakawa, Modern Theory of Polymer Solutions (Harper and Row, New York, 1971).

${ }^{2}$ P. J. Flory, Principles of Polymer Chemistry (Cornell University, Ithaca 1971).

${ }^{3}$ P. G. de Gennes, Scaling Concepts in Polymer Physics (Cornell University, Ithaca, 1979).

${ }^{4} \mathrm{~J}$. Des Cloizeaux, Phys. Rev. A 10, 1665 (1974).

${ }^{5} J$. Des Cloizeaux, J. Phys. (Paris) 42, 5 (1981).

${ }^{6}$ L. Schäfer, and T. A. Witten, J. Chem. Phys. 66, 2121 (1977).

${ }^{7}$ D. J. Elderfield, J. Phys. C 13, 5883 (1980).

${ }^{8} \mathrm{Y}$. Oono, T. Ohta, and K. F. Freed, J. Chem. Phys. 74, 6458 (1981).

${ }^{9}$ A. R. Khokhlov, Makromol. Chem. Rapid Commun. 2, 633 (1981).

${ }^{10}$ P. J. Flory and W. R. Krigbaum, J. Chem. Phys. 18, 1086 
(1950); A. Isihara and R. Koyama, J. Chem. Phys. 25, 712 (1956); E. F。Casassa, Polymer 3, 625 (1962).

${ }^{11} \mathrm{~J}$. L. Martin, M. F. Sykes, and F. T. Hioe, J. Chem. Phys. 46, 3478 (1967).

${ }^{12}$ T. A. Witten, J. Chem. Phys. 76, 3300 (1982).

${ }^{13} \mathrm{D}$. MeIntyre, J. Mazur, and A. M. Wims, J. Chem. Phys. 49,2887 (1968).

${ }^{14} J$. Des Cloizeaux, J. Phys. (Paris) 41, 223 (1980).

${ }^{15}$ M. Dauod and J. P. Cotton, Saclay preprint (1982).

${ }^{16}$ The exponent analogous to $A_{4}$ for a grand ensemble of branch lengths $\mathrm{N}_{1} \cdots \mathrm{N}_{4}$ is known, and is related to the correctionsto-scaling exponent $\omega$ described by E. Brézin, J. C. Le Guillou, and J. Zinn-Justin, in Phase Transitions and Critical Phenomena, edited by C. Domb and M. S. Green (Academic, New York, 1976), Vol. 6 and E. Brézin, C. De Dominicis and J. Zinn-Justin, Lett. al Nuovo Cimento 9 , 483 (1974). In this ensemble chains of all lengths are pres-

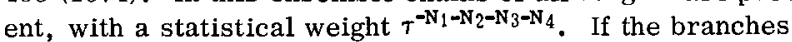
do not interact, their lengths are comparable as $\tau \rightarrow \tau_{c}$ and $\left\langle N_{i}\right\rangle \rightarrow \infty$. But when the branches repel each other this is no longer true. The monomer density $\rho$ increases near the junction point as $r^{1 / v d}$, and is larger by some factor than the density at the same distance from an arbitrary monomer. Thus a monomer saves repulsive energy by moving from the end of a short branch to the end of a long one. The saving is greater if the short branch is shorter. This indicates that a long branch is unstable against growing at the expense of a shorter one. Thus, the stars in the grand ensemble should have almost all their monomers in one branch. The scaling of their partition function thus has no reason to be the same as that for equal-branched stars.
${ }^{17}$ J. C. Le Guillou, and J. Żinn-Justin, Phys. Rev. Lett. 39, 95 (1977).

${ }^{18}$ A. Knoll, L. Schäfer, and T. A. Witten, J. Phys. (Paris) 42, 767 (1981).

${ }^{19} \mathrm{~J}$. Des Cloizeaux, J. Phys. (Paris) 36, 281 (1975).

${ }^{20} \mathrm{P}$. G. De Gennes, talk at Yeshiva meeting on statistical mechanics, New York, Fall 1980.

${ }^{21} \mathrm{~A}$. Baumgartner, Z. Phys. B-Condensed Matter 42, 265 (1981).

${ }^{22}$ Y. Oono and K. F. Freed, J. Phys. A 15, 1931 (1982).

${ }^{23}$ T. A. Witten and L. Schäfer, J. Chem. Phys. 74, 2582 (1981).

${ }^{24}$ We prefer the grand canonical renormalization because it gives a mathematically simpler perturbation expansion than its canonical counterpart. Thus, e.g., the full propagators in the canonical expansion are dominated by a nasty cut, whose structure depends on $d$. Those of the grand canonical expansion are dominated by a simple pole instead. See D. S. McKenzie and M. A. Moore, J. Phys. A 4, 182 (1971). The grand-canonical results have often shown better convergence than their canonical counterparts. See Ref. 23 and J. Prentis, J. Chem. Phys. 76, 1574 (1982).

${ }^{25}$ D. Burch and M. A. Moore, J. Phys. A 9, 435 (1976).

${ }^{26} \mathrm{Y}$. Miyaki, Y. Einaga, and H. Fujita, Macromolecules 11, 1180 (1978).

${ }^{27} \mathrm{~L}$. Schäfer (to be published).

${ }^{28}$ L. Schäfer, Macromolecules 15, 652 (1982).

${ }^{29}$ P. G. De Gennes, J. Chem. Phys. 76, 3316 (1982).

${ }^{30}$ P. G. De Gennes, J. Chem. Phys. 76, 3322 (1982).

${ }^{31} \mathrm{D}$. J. Amit, Field Theory, the Renormalization Group and Critical Phenomena (McGraw- Hill, New York, 1978). 